\title{
NEW METHOD OF USING MOBILE ROBOTS FOR IMPLEMENTING WAREHOUSE OPERATIONS
}

\author{
CBodrenko A., ORCID: 0000-0002-4618-3784, SPIN-code: 8343-3661, Ph.D., \\ Volgograd,Russia,bodrenko@mail.ru
}

\section{НОВЫЙ СПОСОБ ПРИМЕНЕНИЯ МОБИЛЬНЫХ РОБОТОВ ДЛЯ ВЫПОЛНЕНИЯ СКЛАДСКИХ ОПЕРАЦИЙ}

\author{
(СБодренко А. И., ORCID: 0000-0002-4618-3784, SPIN-код: 8343-3661, \\ канд. физ.-мат. наук, г. Волгоград, Россия, bodrenko@mail.ru
}

Abstract. The new patented method of movements of packaged cargoes at the warehouse is proposed in this article. The proposed method of loading is superior in economic efficiency, reliability, cheapness, simplicity of construction and use, to all other methods of loading implemented by robots. The method allows the mobile robot to load a cargo which is three times as heavy as this mobile robot, wherein almost no electrical energy is consumed during loading. In implementing the method, two mobile robots are used, each of which contains a mobile platform, an omnidirectional mobile wheel-type mechanism and a transport suspension with independent suspension for each wheel. Actually, mobile robots help each other to load cargo on the first mobile robot, through robot cooperation. Loading of the container with the load on the first mobile robot is carried out by actuating a two-arm lever, which is carried out through the impact of the bottom of the mobile platform of the second mobile robot on the longer arm of the two-arm lever by lowering the mobile platform of the second mobile robot due to the operation of the transport suspension of the mobile platform of the second mobile robot. For example, if the length of the longer arm of the two-arm lever is three times longer than the shorter arm of the two-arm lever then the second mobile robot of weight $50 \mathrm{~kg}$ is capable of loading a container with a load of weight $150 \mathrm{~kg}$ on the first mobile robot.

Аннотация. В статье представлен новый запатентованный способ перемещения тарноштучных грузов на складе. Этот способ погрузки грузов превосходит по экономической эффективности, надежности, дешевизне, простоте конструкции и использования все другие методы погрузки грузов, осуществляемые роботами. Этот способ позволяет мобильному роботу грузить груз, вес которого в три раза превышает вес этого мобильного робота, при этом во время погрузки почти не расходуется электрическая энергия. При осуществлении способа используются два мобильных робота, каждый из которых содержит мобильную платформу, всенаправленный мобильный механизм колесного типа и транспортную подвеску с обеспечением независимого подвешивания для каждого колеса. Фактически, мобильные роботы помогают друг другу погрузить груз на первый мобильный робот посредством кооперации роботов. Погрузку контейнера с грузом на первый мобильный робот осуществляют посредством приведения в действие двуплечего рычага, которое осуществляют посредством воздействия днища мобильной платформы второго мобильного робота на длинное плечо двуплечего рычага посредством опускания мобильной платформы второго мобильного робота за счет работы транспортной подвески мобильной платформы второго мобильного робота. Например, если длина длинного плеча двуплечего рычага в три 
раза длиннее короткого плеча двуплечего рычага, то второй мобильный робот массой 50 кг может погрузить контейнер с грузом массой 150 кг на первый мобильный робот.

Keywords: mobile robot, mobile platform, two-arm lever, warehouse operations, packaged cargo.

Ключевые слова: мобильный робот, мобильная платформа, двуплечий рычаг, складские операции, тарно-штучный груз.

There are various methods of implementing warehouse operations used by Amazon, Symbotic and other industrial companies.

One of the most important warehouse operations is the loading of a container with a load on a mobile platform. The most widespread method of loading cargo on the mobile robot is implemented by the robot arm. It needs a high-powered machine actuator, which is either very heavy or very expensive, to implement loading of a packaged cargo by using a robot arm. For example, palletizing robot KR 40 PA from KUKA AG has a weight of about $700 \mathrm{~kg}$ and a payload of about $40 \mathrm{~kg}$ (1). In addition, this robot is not a mobile robot and it needs a large amount of electrical energy to load cargoes by this robot. Though the mobile robot KMR iiwa from KUKA AG has a weight of about $420 \mathrm{~kg}$ and a payload of robot arm LBR iiwa $14 \mathrm{R} 820$ of about $14 \mathrm{~kg}$ (2). Though there is an experimental mobile robot, with robot arms, which has a weight of about $110 \mathrm{~kg}$ and a payload of about $45 \mathrm{~kg}$.

For example, Amazon uses the Kiva robot (which has no robot arms) whose weight is about $120 \mathrm{~kg}$, and which costs about $\$ 40000$ (3). The Kiva robot uses heavy and expensive high-powered DC machine actuator which consumes a large amount of electrical energy during the loading of cargo.

Thus, popular methods of implementing warehouse operations require to use rather heavy and expensive mobile robots with a robot arm with relatively low payload, and with high-powered machine actuators which consume too much electrical energy during loading of cargo on the mobile robot.

\section{Merits of the new method of using mobile robots for implementing warehouse operations}

The new method of implementing warehouse operations was invented in June 2018 in (4). This method represented in this article does not require to use high-powered machine actuators. Moreover, according to this method, during the loading of a container with a load on a mobile platform, almost no electrical energy is consumed. This loading is carried out by actuating a twoarm lever. For example, if the length of the longer arm of the two-arm lever is three times longer than the shorter arm of the two-arm lever then the mobile robot of weight $50 \mathrm{~kg}$ is capable of loading a container with a load of weight $150 \mathrm{~kg}$ on another mobile robot.

The new method relates to the field of robotics and to warehouses of enterprises and shops. Method of movement of packaged goods in a warehouse includes intra-warehouse movements of goods placed in containers containing handles, loading a container loaded onto a mobile robot, moving a container loaded onto a mobile robot, unloading the container loaded onto a mobile robot. In implementing the method, two mobile robots are used, each of which contains a mobile platform, an omnidirectional mobile wheel-type mechanism and a transport suspension with independent suspension for each wheel. According to the new method, a two-arm lever and a ratchet mechanism are installed on the mobile platform of the first mobile robot. When loading a container with a load on the first mobile robot, mobile robots are positioned so that the bottom of the mobile platform of the second mobile robot is located above the long arm of the two shoulders lever, and a hook 
located at the end of the short arm of the two shoulders lever is placed in the space between the upper part of the container with the load and the handle of this container. Loading of the container with the load on the first mobile robot is carried out by actuating a two-arm lever, which is carried out through the impact of the bottom of the mobile platform of the second mobile robot on the long arm of the two shoulders lever by lowering the mobile platform of the second mobile robot due to the operation of the transport suspension of the mobile platform of the second mobile robot. Moving the container with the cargo is carried out on the first mobile robot.

Technical result, the achievement of which the new method is directed, is to ensure the loading of packaged cargoes on a mobile robot and their movement on a mobile robot, as well as unloading of packaged cargoes from a mobile robot.

Two mobile robots used to carry out the new method of implementing warehouse operations

The first mobile robot is used for loading, carrying and unloading of the packaged cargoes, and the second mobile robot is used for loading of the packaged cargoes on the first mobile robot (through the implementation of automatic operations of these mobile robots). Each mobile robot, which is a wheeled robot, comprises: mobile platform having an omnidirectional mobile mechanism with brake system; onboard computer with information storage device; Wi-Fi network node equipment connected to the onboard computer; electric battery; two video cameras; two supports on which two video cameras are installed. The brake systems, installed on mobile robots, support stability of these robots during picking of the packaged cargo from the storage place.

The load capacity of the first mobile robot is more than $200 \mathrm{~kg}$. The T-shaped two-arm lever with end-of-travel stop and electronically controlled ratchet mechanism are installed on the mobile platform of the first mobile robot. Electronically controlled ratchet mechanism, when it is switched on, prevents the shorter arm of the two-arm lever form lowering. The length of the longer arm of the two-arm lever is more than $120 \mathrm{~cm}$. The length of the shorter arm of the two-arm lever is from 35 to $40 \mathrm{~cm}$. The weight of the shorter arm of the two-arm lever is more than $2 \mathrm{~kg}$. The weight of the longer arm of the two-arm lever is less than $400 \mathrm{~g}$. The shorter arm of the two-arm lever has a hookshaped end by which the packaged cargo is picked. The first mobile robot has a geometry-stabilized mobile platform, deriving its stability from its shape, and therefore the first mobile robot can be very light. Thus, the mobile platform of the first mobile robot is made from deformation-resistant, strong, lightweight material. The first mobile robot is represented in Figure 1.

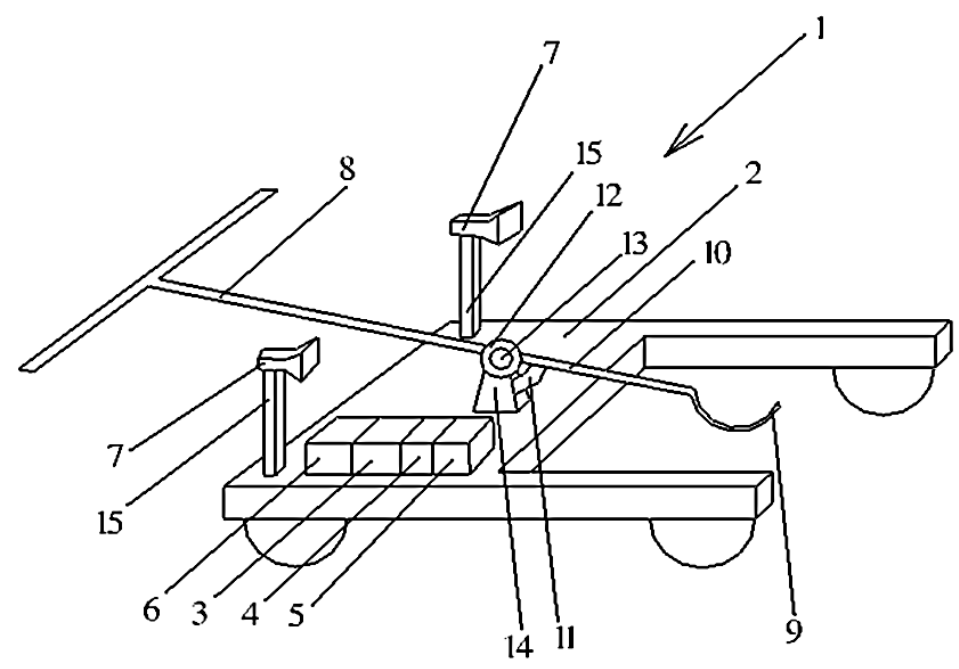

Figure 1. The view of the first mobile robot. 
Each mobile platform includes omnidirectional mobile mechanism, with an electronically controlled brake system, which comprises the set of actuators and proprioceptive sensors which are encoders.

The weight of the second mobile robot is from 25 to $50 \mathrm{~kg}$. The width and length of the mobile platform of the second mobile robot are from 100 to $150 \mathrm{~cm}$. Electronically controlled, height-adjustable independent suspension with independent suspension for each wheel is installed on the mobile platform of the second mobile robot. The height-adjustable independent suspension provides raising and lowering of the mobile platform of the second mobile robot.

The second mobile robot is represented in Figure 2.

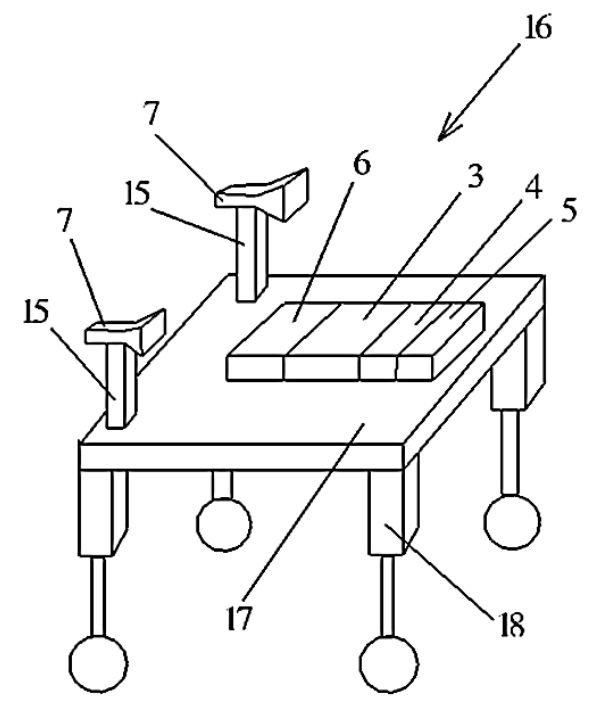

Figure 2. The view of the second mobile robot.

On Figures 1 and 2, the following items were denoted by the numbers: 1 - the first mobile robot, 2 - the mobile platform of the first mobile robot, 3 - onboard computer, 4 - information storage device connected to the onboard computer, 5 - network node equipment connected to the onboard computer, 6 - electric battery, 7 - video camera, 8 - the longer arm of the two-arm lever, 9 - the hook, 10 - the shorter arm of the two-arm lever, 11 - the end-of-travel stop of the two-arm lever, 12 - the ratchet mechanism, 13 - axis on which the two-arm lever and the ratchet mechanism are installed, 14 - support on which the axis is installed, 15 - support on which the video camera is installed, 16 - the second mobile robot, 17 - the mobile platform of the second mobile robot, 18 - element of the height-adjustable independent suspension.

The two-arm lever is made from deformation-resistant, strong, hard, lightweight material. The end-of-travel stop of the two-arm lever prevents the shorter arm of the two-arm lever from lowering when the ratchet mechanism is switched off, due to the fact that the shorter arm of the two-arm lever weighs four times heavier than the longer arm of the two-arm lever.

Each video camera is capable of getting and transferring the images to the onboard computer of the mobile robot, on which this video camera is installed, for image processing at $30 \mathrm{fps}$ for $1024 \times 1024$ pixels. Each onboard computer is capable of real-time image processing of the input images received from two video cameras (at $30 \mathrm{fps}$ for $1024 \times 1024$ pixel, for each video camera). The real-time image processing is done by using software for computer vision systems, Linux-based real-time operating system, software for parallel computing, and software allowing applications to run on real-time operating systems. Thus, the onboard computer and two video cameras connected to it form a real-time vision system. 
The electric battery provides electric power to the mobile robot on which this electric battery is installed.

The ratio of the weight of the container with the packaged cargo to the weight of the second mobile robot is less than the ratio of the length of the longer arm of the two-arm lever to the length of the shorter arm of the two-arm lever. The load capacity of the first mobile robot is more than the total weight of the second mobile robot and the container with the packaged cargo.

\section{Software used to carry out the new method of implementing warehouse operations}

A real-time operating system (RTOS) is installed on the onboard computers of the first and the second mobile robots, and on the computer of the control center. Thus, the following RTOS is installed: NI Linux Real-Time (5).

In order to implement automatic operations of mobile robots, the following software is installed on the onboard computers of the first and the second mobile robots, and on the computer of the control center:

-LabVIEW Robotics Bundle (6);

-The Orocos Real-Time Toolkit (7);

-OpenCV software (Open Source Computer Vision Library) (8) with using standard OpenVX (9) and with using the CUDA technology (Compute Unified Device Architecture) (10);

-OpenCL software (Open Computing Language) (11);

-OpenMP software (Open Multi-Processing) (12) and OpenMP compilers (13);

-the NTPD program (Network Time Protocol daemon) (14).

NI Linux Real-Time is a Linux-based real-time OS, fully supported in by the NI LabVIEW development environment with the LabVIEW Real-Time Module.

LabVIEW Robotics Bundle includes the software tools needed to control a mobile robot.

The Orocos Real-Time Toolkit provides a $\mathrm{C}++$ framework aiming the implementation of realtime control systems. The Orocos Real-Time Toolkit allows the software to run on real-time operating systems.

OpenCV includes software for computer vision systems with a focus on real-time applications.

OpenVX is an open standard for cross platform acceleration of computer vision software. OpenVX allows power-optimized computer vision processing, especially important in embedded and real-time use cases such as smart video surveillance, object and scene reconstruction, augmented reality, visual inspection, robotics (9).

CUDA is a parallel computing platform from NVIDIA for general purpose computing on graphical processing units (GPUs).

OpenCL is the open standard for cross-platform, parallel programming of diverse compute devices, including GPUs.

OpenMP includes API that supports multi-platform shared-memory parallel programming in $\mathrm{C} / \mathrm{C}++$. The OpenMP API is used for developing parallel applications on various platforms.

The Network Time Protocol daemon (NTPD) is used in order to maintain the system time on the onboard computers of the first and the second mobile robots in synchronization with a time server using the Network Time Protocol (NTP). The computer of the control center is used as a time server. 


\section{Loading of the container with the load on the first mobile robot through robot cooperation}

The weight of a packaged cargo to be carried is from 1 to $150 \mathrm{~kg}$ while the packaged cargo dimensions are from 25 to $50 \mathrm{~cm}$ (width, length, height). Each packaged cargo is placed into the container having a handle. The container dimensions are from 60 to $70 \mathrm{~cm}$ (width, length, height) while the weight of the container is less than $2 \mathrm{~kg}$.

Localization of each mobile robot is conducted continuously (by using the software installed on the onboard computer of this mobile robot) through the dead reckoning by using sensor fusion and analyzing information received from video cameras and proprioceptive sensors of this robot.

At first, the name of the packaged cargo to be transported is inputted into the computer of the control center. Then the storage place, where this packaged cargo is stored in the container, is found on the computer of the control center. After that, the first and the second mobile robots receive (on the onboard computers) the parameters of the storage place and the name of packaged cargo to be transported, and move to this storage place. The first mobile robot takes a pose in which the hookshaped end of the shorter arm of the two-arm lever is placed between the handle of the container and the upper part of this container. Then the second mobile robot takes a pose in which the mobile platform of the second mobile robot is posed (by the work of the height-adjustable independent suspension) above the longer arm of the two-arm lever. After that, in order to maintain the stability of the robots, the brake systems of the mobile mechanisms are activated, and the ratchet mechanism installed on the mobile platform of the first mobile robot is switched on.

The poses of the first and the second mobile robots at the moment before the beginning of the loading of cargo are represented on Figure 3.

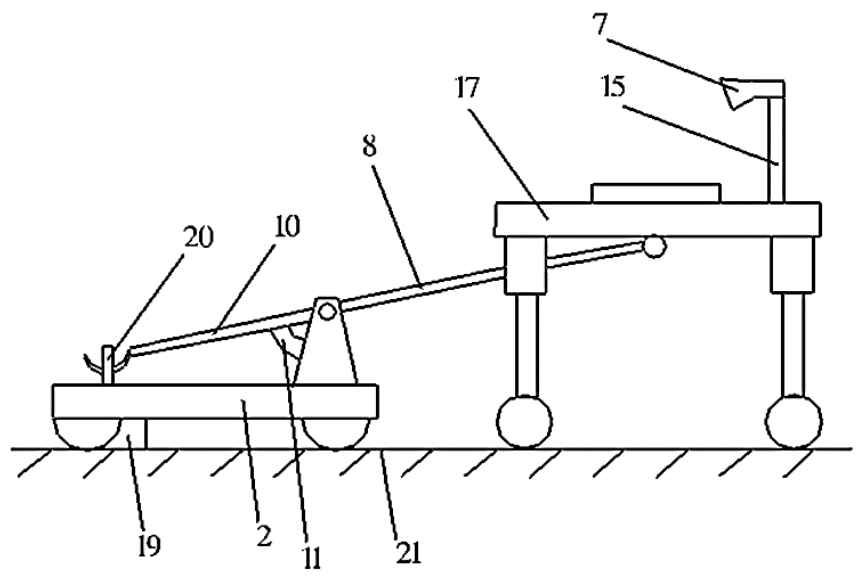

Figure 3. The side view of the first and the second mobile robots at the moment before the beginning of picking of the packaged cargo from the storage place.

After that, the mobile platform of the second mobile robot is lowered by the work of the height-adjustable independent suspension. Then the mobile platform of the second mobile robot pressures on the longer arm of the two-arm lever, and the shorter arm of the two-arm lever moves upward while the handle of the container is hitched by the hook. Wherein the ratchet mechanism prevents the shorter arm of the two-arm lever from lowering, and therefore, prevents the container with the packaged cargo from lowering because the ratchet mechanism was switched on. The Tshaped longer arm of the two-arm lever provides stability of the second mobile robot during loading of the container on the first mobile robot. Thus the container with the packaged cargo is loaded on the first mobile robot.

After that, the mobile platform of the second mobile robot is raised by the height-adjustable independent suspension. Then the brake systems of the mobile mechanisms are deactivated. Then 
the second mobile robot moves to the parking place while the first mobile robot moves to the picking area of this warehouse and unloads the container with the packaged cargo through the implementation of automatic operations of this mobile robot.

Unloading of packaged cargo from the first mobile robot is provided by switching off the ratchet mechanism. After the ratchet mechanism is switched off, the shorter arm of the two-arm lever moves downward and the container with the packaged cargo is placed into the storage container for packaged cargoes transported to the picking area. This completes the intra-warehouse movements of the container with the packaged cargo from the storage area to the picking area in the warehouse.

The poses of the first and the second mobile robots at the moment after loading of the container with the load on the first mobile robot through robot cooperation are represented in Figure 4.

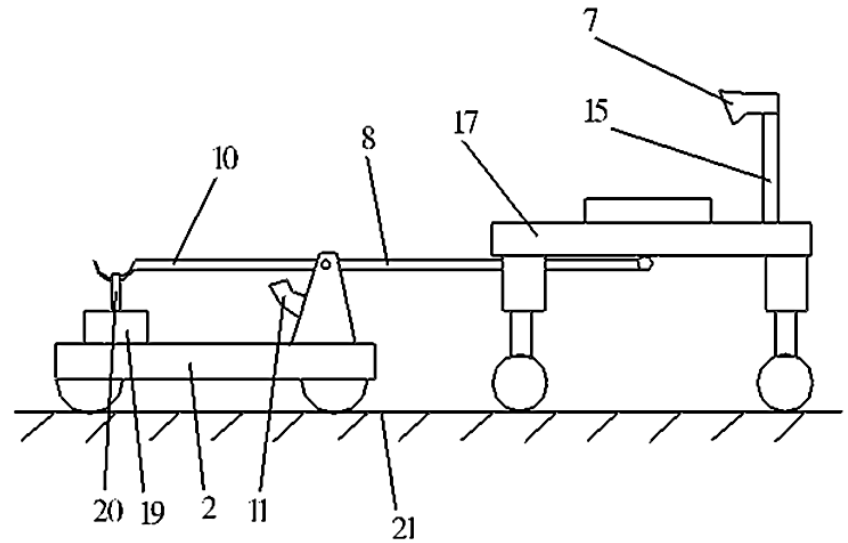

Figure 4. The side view of the first and the second mobile robots at the moment after picking of the packaged cargo from the storage place.

On the Figures 3 and 4, the following items were denoted by the numbers: 2 - the mobile platform of the first mobile robot, 7 - video camera, 8 - the longer arm of the two-arm lever, 10 - the shorter arm of the two-arm lever, 11 - the end-of-travel stop of the two-arm lever, 15 support on which the video camera is installed, 17 - the mobile platform of the second mobile robot, 19 - container, 20 - handle of the container, 21 - the floor of the warehouse building.

\section{The warehouse management system}

The warehouse building is one story. The warehouse has a storage area and a picking area not intersecting with each other. At the warehouse, the containers having handles loaded with the packaged cargoes are located on the floor in the storage places in the storage area.

The warehouse aisle intended for movements of the first and the second mobile robots, during implementing warehouse operations, is established at the warehouse.

The control center is located in the warehouse and outside the storage area, the picking area, and the warehouse aisle intended for movements of the first and the second mobile robots. The control center comprises computer with information storage device; Wi-Fi network node equipment connected to the computer of the control center.

The plan of the warehouse is represented in Figure 5. 


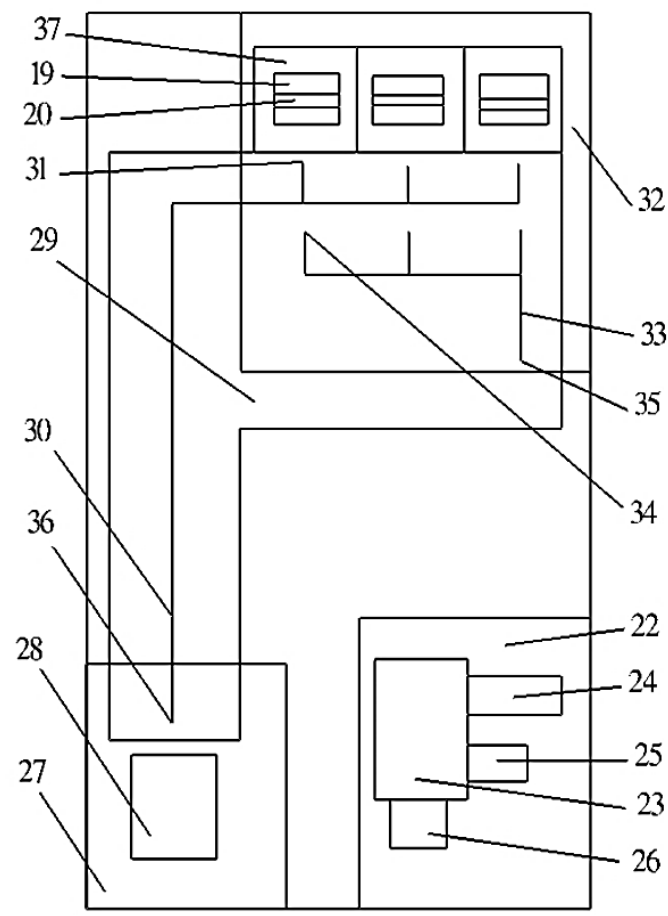

Figure 5. The plan of the warehouse.

On Figure 5, the following items were denoted by the numbers: 19 - container, 20 - handle of the container, 22 - the control center, 23 - computer of the control center, 24 - information storage device connected to the computer of the control center, 25 - network node equipment connected to the computer of the control center, 26 - input device connected to the computer of the control center, 27 - the picking area, 28 - storage container for packaged cargoes transported to the picking area, 29 - the warehouse aisle intended for movements of the first and the second mobile robots, 30 - line of possible movements of the mobile platform of the first mobile robot, 31 - orthogonal projection of the mobile platform reference point (of the first mobile robot) on the plane OXY of the world coordinate system during picking of the packaged cargo from the storage place, 32 - the storage area, 33 - line of possible movements of the mobile platform of the second mobile robot, 34 - orthogonal projection of the mobile platform reference point (of the second mobile robot) on the plane OXY of the world coordinate system during picking of the packaged cargo from the storage place, 35 - orthogonal projection of the mobile platform reference point (of the second mobile robot) on the plane OXY of the world coordinate system during parking of the second mobile robot, 36 - orthogonal projection of the mobile platform reference point (of the first mobile robot) on the plane OXY of the world coordinate system during unloading of the packaged cargo from the first mobile robot, 37 - storage place.

The world coordinate system OXYZ is orthogonal, right-oriented, and the world coordinate system origin is on the upper surface of the floor of the warehouse building. In addition, the plane OXY contains the upper horizontal surface of the floor of the warehouse building, the axis OZ is directed vertically upward and perpendicularly to the plane OXY. The axes OX and OY are mutually perpendicular and belong to the plane OXY.

For each video camera, the coordinate system of the video camera is introduced. The coordinate system of the video camera is orthogonal, right-oriented, and the unit vector, giving the direction of the third coordinate axis of this coordinate system, is on the optical axis of this video camera, and directed towards the video object, and the unit vector, giving the direction of the first 
coordinate axis of this coordinate system, is orthogonal to the unit vector, giving the direction of the third coordinate axis of this coordinate system. In addition, the origin of the coordinate system of the video camera is on the surface of the video camera body, at the point of intersection of this surface and the optical axis of this video camera.

For each mobile platform, the coordinate system of the mobile platform is introduced. The coordinate system of the mobile platform is orthogonal, right-oriented, and the unit vector, giving the direction of the first coordinate axis of this coordinate system, is directed in the forward direction of this mobile platform, and the unit vector, giving the direction of the third coordinate axis of this coordinate system, is directed vertically upward from the mobile platform, and the origin of the coordinate system of the mobile platform is the mobile platform reference point which is on the upper surface of this mobile platform.

These parameters of the world coordinate system, the coordinate systems of each video camera, the coordinate systems of each mobile platform are obtained by using a 3D scanner and stored on the computer of the control center and on the onboard computers of the mobile robots.

The parameters of the pose of each video camera, at the moment of time, are ordered set of nine numbers, where the first three numbers are the coordinates of the origin of the coordinate system of the video camera calculated in the world coordinate system, the next three numbers are the coordinates of the unit vector which is on the optical axis of this video camera, and directed towards the video object, calculated in the world coordinate system, the other three numbers are the coordinates of the unit vector, giving the direction of the first coordinate axis of the coordinate system of this video camera, calculated in the world coordinate system. These parameters stored on the computer of the control center and on the onboard computers of the mobile robots.

The parameters of the pose of each mobile platform of the mobile robot, at the moment of time, are ordered set of six numbers, where the first three numbers are the coordinates of the origin of the coordinate system of this mobile platform calculated in the world coordinate system, the other three numbers are the coordinates of the unit vector, giving the direction of the first coordinate axis of the coordinate system of this mobile platform, calculated in the world coordinate system. These parameters stored on the computer of the control center and on the onboard computers of the mobile robots.

The parameters of the pose of each mobile platform, at the moment of time, are obtained through the dead reckoning by using sensor fusion and analyzing information received from video cameras and proprioceptive sensors of this robot, and by using:

-data, received by an onboard computer, in the form of signals from the proprioceptive sensors installed on this mobile platform;

- the parameters of the poses of this mobile platform, at the previous moments of time, and which were obtained before;

-the parameters of the poses of the two video cameras installed on this mobile platform calculated both in the world coordinate system and the coordinate system of this mobile platform;

-images received from the two video cameras installed on this mobile platform, and software for computer vision, installed on the onboard computer of this mobile robot.

The warehouse aisle intended for movements of the first and the second mobile robots is established in the form of an oriented simple two-dimensional polygon located on the plane OXY of the world coordinate system by setting the vertices of a simple broken-line which is the boundary of this polygon. This simple broken-line is established by an ordered set of numbers which are the coordinates of the vertices of this simple broken-line, calculated in the world coordinate system, and written in accordance with the traversal order of the vertices of this simple two-dimensional polygon. This ordered set of numbers is the parameters of the warehouse aisle. 
The lines of possible movements of the mobile platform of the first and the second mobile robots are established in the form of broken-lines located on the plane OXY of the world coordinate system, which are the lines of possible movements of the orthogonal projections of the mobile platform reference point on the plane OXY of the world coordinate system during movements of this mobile platform.

Each such broken-line is established by an ordered set of numbers which are the coordinates of the vertices of this simple broken-line, located on the plane OXY of the world coordinate system, and calculated in the world coordinate system, and written in accordance with the traversal order of the vertices of this broken-line. This ordered set of numbers is parameters of this line of possible movements of the mobile platform of the mobile robot.

The parameters of the warehouse aisle and the parameters of the lines of possible movements of the mobile platforms of the mobile robots are obtained by using a 3D scanner and stored on the computer of the control center and on the onboard computers of the mobile robots.

For each storage place on the warehouse floor, the following parameters of the storage place (in items form (a) to (g)) are obtained:

(a) the address of the storage place by which this storage place can be uniquely found in this warehouse;

(b) the coordinates of the four points located on the upper surface of the warehouse floor, calculated in the world coordinate system, that determine the largest in area rectangle, which can be located on this storage place;

(c) the coordinates of the point, calculated in the world coordinate system, located on the upper surface of the warehouse floor, and which is set so that the orthogonal projection of the origin of the coordinate system of the container, stored in this storage place, coincides with this point;

(d) the parameters of the pose of the mobile platform of the first mobile robot, at the moment of time right before beginning of picking of the packaged cargo from this storage place (when the hook is placed between the handle of the container and the upper part of this container), i. e. the coordinates of the origin of the coordinate system of the mobile platform, calculated in the world coordinate system, and the coordinates of the unit vector, giving the direction of the first coordinate axis of the coordinate system of this mobile platform, calculated in the world coordinate system;

(e) the parameters of the poses of the two video cameras installed on the mobile platform of the first mobile robot, such that the images received through these two video cameras, at the moment of time right before the beginning of picking of the packaged cargo from this storage place, contain the view of this storage place;

(f) the parameters of the pose of the mobile platform of the second mobile robot, at the moment of time right before beginning of picking of the packaged cargo from this storage place (when the bottom of the mobile platform of the second mobile robot is located above the longer arm of the two-arm lever), i. e. the coordinates of the origin of the coordinate system of this mobile platform calculated in the world coordinate system, and the coordinates of the unit vector, giving the direction of the first coordinate axis of the coordinate system of this mobile platform, calculated in the world coordinate system;

(g) the parameters of the coordinate system of this storage place, i. e. the coordinates of the origin of this coordinate system, calculated in the world coordinate system, and the coordinates of the three unit vectors giving the directions of the three coordinate axes of this coordinate system, calculated in the world coordinate system.

Before the packaged cargoes are located in the storage area, each packaged cargo is placed in a container having a handle. Each packaged cargo is rigidly fixed inside the container so that the 
center of mass of this packaged cargo is located closest to the center of mass of this container. Only identical containers are used.

For each container, the following parameters are obtained:

-the name of the packaged cargo located in this container;

-the total weight of the container and the packaged cargo located in this container;

-the parameters of the coordinate system of this container, wherein the origin of this coordinate system coincides with the center of mass of this container;

-the parameters of the 3D-model of this container.

After that, the containers with the packaged cargoes are placed in the storage places. Wherein each container is placed so that the handle is positioned orthogonally to the forward direction of the mobile platform of the first mobile robot during loading of this container on the first mobile robot, at the moment of time right before the beginning of picking of the packaged cargo from this storage place. Then the information on names of the packaged cargoes, and addresses of the storage places where these packaged cargoes are placed, are stored on the computer of the control center and on the onboard computers of the mobile robots. After that, the 3D-model of this warehouse building, with the warehouse building's contents, is obtained by using a 3D scanner. The parameters of this 3D-model are stored on the computer of the control center and on the onboard computers of the mobile robots.

\section{Conclusion}

The proposed method of loading of cargo on a mobile robot is superior in economic efficiency, reliability, cheapness, simplicity of construction and use, to all other methods of loading implemented by robots. This method derives its superiority from the using of the two-arm lever by two mobile robots helping each other to load cargo, through the implementation of automatic operations of these mobile robots. The proposed method does not require to use heavy loading equipment and high-powered machine actuators. The first mobile robot can be very light, but with high load capacity. The weight of the second mobile robot depends on the weight of cargo to be loaded, and on the lengths of shorter and longer arms of the two-arm lever. Moreover, almost no electrical energy is consumed during the loading of cargo by using this method.

The proposed method of implementing warehouse operations can be used in various warehouses of large industrial companies as well as in warehouses of small manufacturing companies. Therefore the proposed method of implementing warehouse operations can be used in various industries, such as the construction industry, the retail industry, etc.

\section{Sources:}

(1). KR 40 PA. Available at: https://clck.ru/Frh3E, accessed 14.04.2019.

(2). KMR iiwa. Available at: https://clck.ru/Frh3R, accessed 14.04.2019.

(3). Is Kiva systems is a good fit for your distribution center? An unbiased distribution consultation evaluation. Available at: https://clck.ru/Frh3b, accessed 14.04.2019.

(4). Bodrenko, A. I. (2019). Method of movement of tarno-piece goods in the storage. Patent RU2681471C1. Available at: https://clck.ru/Frh3h, accessed 14.04.2019.

(5). Introduction to NI Linux Real-Time. Available at: https://clck.ru/Frh3p, accessed 14.04.2019.

(6). Overview of the LabVIEW Robotics Module. Available at: https://clck.ru/Frh3u, accessed 14.04.2019.

(7). The Orocos Real-Time Toolkit. Available at: http://www.orocos.org/rtt, accessed 14.04.2019. 
(8). Open Source Computer Vision Library. Available at: http://opencv.org, accessed 14.04.2019.

(9). OpenVX Overview. Available at: http://www.khronos.org/openvx/, accessed 14.04.2019.

(10). CUDA Zone. Available at: https://developer.nvidia.com/cuda-zone, accessed 14.04.2019.

(11). OpenCL Overview. Available at: https://www.khronos.org/opencl/, accessed 14.04.2019.

(12). The OpenMP API specification for parallel programming. Available at: http://www.openmp.org/, accessed 14.04.2019.

(13). OpenMP compilers \& Tools. Available at: https://clck.ru/Frh46, accessed 14.04.2019.

(14). Network Time Protocol daemon. Available at: http://doc.ntp.org/4.1.0/ntpd.htm, accessed 14.04.2019.

Работа поступила

в редакцию 14.04.2019 2.
Принята к публикации 20.04.2019 2.

Ссылка для циитирования:

Bodrenko A. New Method of Using Mobile Robots for Implementing Warehouse Operations // Бюллетень науки и практики. 2019. Т. 5. №5. С. 221-232. https://doi.org/10.33619/2414$2948 / 42 / 30$

Cite as $(A P A)$ :

Bodrenko, A. (2019). New Method of Using Mobile Robots for Implementing Warehouse Operations. Bulletin of Science and Practice, 5(5), 221-232. https://doi.org/10.33619/24142948/42/30. 